\title{
Morphology of colorectal lymphoid aggregates in cancer, diverticular and inflammatory bowel diseases
}

\author{
Riccardo Nascimbeni ${ }^{1}$, Francesco Di Fabio ${ }^{1}$, Ernesto Di Betta ${ }^{1}$, Pierpaolo Mariani ${ }^{1}$, \\ Simona Fisogni ${ }^{2}$ and Vincenzo Villanacci ${ }^{2}$ \\ ${ }^{1}$ Cattedra di Chirurgia Generale of the University of Brescia, Brescia, Italy and ${ }^{2}$ Cattedra di Anatomia \\ Patologica of the University of Brescia, Brescia, Italy
}

\begin{abstract}
The present study compares the characteristics of colorectal lymphoid aggregates in patients with carcinoma, diverticular disease, Crohn's disease, or ulcerative colitis of the large bowel. A total of 77 patients (41 colorectal cancer, 27 diverticular disease, six ulcerative colitis, three Crohn's disease) undergoing colorectal resection were included. Acetic acid staining, hematoxylin and eosin staining, CD3, CD20, and MIB1 immunostaining were employed in order to assess density, diameter, subepithelial or basal location, cellular profile, and proliferation of lymphoid aggregates in normal-appearing and actively inflamed large bowel. In normalappearing tissue, mean density of lymphoid aggregates was lower in patients with ulcerative colitis and Crohn's disease than in those with colorectal cancer or diverticular disease. A larger mean diameter of aggregates was observed in patients with Crohn's disease. In inflammatory bowel diseases, a marked increase of the mean density of lymphoid aggregates was observed in actively affected specimens. In Crohn's disease more than in ulcerative colitis, the aggregates had a predominant basal or transmural distribution. In diverticular disease, active inflammation determined a less significant increase of subepithelial aggregates harboring a lower proportion of germinal centers. No significant variations of CD3, CD20, and MIB1 were recorded among the four disease groups. The lymphoid aggregate derangements observed not only in the actively affected mucosa but also in the unaffected colorectal lining of patients with Crohn's disease and ulcerative colitis support a relevant involvement of lymphoid aggregate system in the pathogenesis of inflammatory bowel diseases.
\end{abstract}

Modern Pathology (2005) 18, 681-685, advance online publication, 3 December 2004; doi:10.1038/modpathol.3800343

Keywords: colorectal cancer; Crohn's disease; diverticular disease; lymphoid aggregates; ulcerative colitis

The morphologic modifications of colorectal lymphoid aggregates in response to intestinal diseases have been scarcely investigated. Even their frequency in healthy individuals is obscure, and, since the pioneeristic study of Dukes and Bussey, ${ }^{1}$ a wide range of values has been reported., ${ }^{2,3}$

Although sporadic papers have described the lymphoid aggregate features in several large bowel diseases ${ }^{2,4-6}$ their pathogenic role in common colorectal diseases is largely unexplored. Nonetheless, a transmural distribution of lymphoid aggregates is considered a histological peculiarity of Crohn's disease, ${ }^{7}$ and the clinical implementation of lymphoid aggregate analysis has been proposed, as they

Correspondence: Dr R Nascimbeni, MD, Cattedra di Chirurgia Generale, Università degli Studi di Brescia, Via Pila 3, 25135 Brescia, Italy.

E-mail: nascsurg@hotmail.com

Received 10 May 2004; revised 8 October 2004; accepted 10 October 2004; published online 3 December 2004 may be detected both on double-contrast barium enema $^{8}$ and on colonoscopy. ${ }^{9}$

The aim of the present study was to compare the morphologic characteristics of lymphoid aggregates in the colon and the rectum of patients undergoing colonic resection for carcinoma, inflammatory bowel disease, or diverticular disease.

\section{Materials and methods}

A total of 77 consecutive patients undergoing colorectal resection were included in the study. Indication for surgery was: sporadic colorectal cancer in 41 subjects, diverticular disease in 27 subjects, ulcerative colitis in six subjects, and Crohn's disease in three subjects.

All patients with inflammatory bowel disease underwent surgery due to clinically and histologically severe disease involving the left colon for Crohn's disease, and the left colon and the rectum 
for ulcerative colitis. None of the cases showed an adequate response to medical therapy.

Surgical specimens were opened and washed with cold isotonic solution immediately after resection. Strips of normal-appearing mucosa and submucosa were dissected from the underlying muscularis propria according to a standard topographic scheme. The area of each strip was calculated and recorded.

Lymphoid aggregates were visualized using the method developed by Langman and Rowland, ${ }^{3}$ modified as follows. The strips were immersed in a $2.5 \%$ acetic acid solution for $100 \mathrm{~min}$ and then they were fixed in $10 \%$ buffered formalin. Subsequently they were placed, luminal side up, on a glass slide and examined with a light microscope at $\times 10$ magnification by a single observer, who was blinded as to colorectal disease and colonic site. Lymphoid aggregates, visible as dark patches, were counted and their diameter measured using a micrometric optical device. In this phase, no strip was collected from colorectal segments actively involved by the inflammatory process, since architectural derangement hampered an adequate evaluation with the acetic acid staining method.

In order to validate the staining method described above, the entire measurement process was performed in 50 putative lymphoid aggregates which were microdissected with the overlying mucosa along with 50 comparable samples of normalappearing colorectal wall from the surrounding bowel tissue. Each specimen was stained with hematoxylin and eosin (H\&E) and observed by a single pathologist unaware of the sample group.

One additional mucosal strip $(2 \mathrm{~cm} \times 1 \mathrm{~cm})$ of normal-appearing colon or rectum was obtained from each surgical specimen, and one further strip $(2 \mathrm{~cm} \times 1 \mathrm{~cm})$ was collected from each surgical specimen with diverticular or inflammatory bowel diseases in areas with macroscopical signs of inflammation. Each strip was fixed in $10 \%$ buffered formalin and paraffin embedded. Serial $2-\mu \mathrm{m}$ sections were examined histologically after routine H\&E staining. The following parameters were assessed: lymphoid aggregate density, and rate of aggregates with visible germinal centers, and rate of extramucosal or basal aggregates.

From the same tissue blocks, further $5-\mu \mathrm{m}$ sections were obtained and stained with monoclonal mouse antibody against the intracytoplasmic epithope of CD20 protein (DakoCytomation, Glostrup, Denmark; dilution 1:100) to demonstrate B cells, and with monoclonal mouse antibody against the $20 \mathrm{kDa}$ band corresponding to $\mathrm{CD} 3_{\varepsilon}$ (DakoCytomation dilution 1:40) to demonstrate T cells.

Cellular proliferation was evaluated using monoclonal mouse antibody against native Ki67 protein (MIB-1) (DakoCytomation dilution 1:50). The lymphocyte proliferation index was the rate of nuclear-positive cells on total cell number for each lymphoid aggregate.

\section{Statistical Analysis}

Normal distribution of the frequency and diameter of lymphoid aggregates was assessed using graphical methods and Shapiro-Wilk test. Data were logtrasformed when necessary for variance stabilizing. Original data are shown in tables for better comprehension. One-way analysis of variance with the Bonferroni adjustment was performed to measure difference of LA frequencies and diameters in patients with colorectal cancer, diverticular disease and inflammatory bowel disease, and among different tumor stages. The $t$-test was used to assess the difference of frequencies and diameters according to age, gender, tumor location (right vs left large bowel; colon vs rectum), and between neoplastic and diverticular sigmoid colon. The analysis of the histological and immunohistochemical variables (aggregates with germinal centers, basal aggregates, CD3- and CD20-positive aggregates, MIB-1 index) was carried out using the nonparametric tests of Mann-Whitney and Kruskal-Wallis, as their values' distribution was skewed. The level for statistical significance was set at $\alpha=0.05$ and confidence intervals were determined at the 95\% level. All analyses were performed using statistical software (Stata version 7.0 for Windows, Stata Corporation; College Station, TX, USA).

\section{Results}

The median age of patients was 68 years (range 41-91 years) in colorectal cancer group, 63 years (range 32-83 years) in diverticular disease group, and 49 years (range 22-73 years) in patients with inflammatory bowel disease.

The male-female ratio of subjects with colorectal cancer, diverticular disease, and inflammatory bowel disease was 1.4, 0.4, and 0.5, respectively.

\section{Acetic Acid Staining}

A total of 438 mucosa and submucosa strips were collected and stained with acetic acid for a total area of $5249.3 \mathrm{~cm}^{2}$. The results of the subsequent lymphoid aggregate count and diameter measurement are summarized in Table 1.

As stated previously, assessments with acetic acid staining were performed on normal-appearing mucosa only. The method was validated by confirming histologically the presence of an aggregate in 49 out of the 50 dark patches microdissected. Among the 50 control samples, none contained a lymphoid aggregate.

The mean frequency of lymphoid aggregates did not vary according to patient age or gender.

The lymphoid aggregate frequency (Figure 1) varied significantly among the four disease groups, being higher in the mucosa of patients with colorectal cancer or diverticular disease than in the 
Table 1 Overview of lymphoid aggregate features (acetic acid staining)

\begin{tabular}{|c|c|c|c|c|}
\hline & $\begin{array}{c}\text { Colorectal } \\
\text { cancer }\end{array}$ & $\begin{array}{c}\text { Diverticular } \\
\text { disease }\end{array}$ & $\begin{array}{c}\text { Ulcerative } \\
\text { colitis }\end{array}$ & $\begin{array}{l}\text { Crohn's } \\
\text { disease }\end{array}$ \\
\hline Specimen no. & 41 & 27 & 6 & 3 \\
\hline Mean lymphoid aggregate density (aggregate count/ $\mathrm{cm}^{2} \pm$ s.d.) & $2.7 \pm 1.7$ & $2.1 \pm 1.0$ & $0.7 \pm 0.7$ & $0.3 \pm 0.2$ \\
\hline Lymphoid aggregate diameter $(\mu \mathrm{m} \pm$ s.d.) & $800 \pm 150$ & $610 \pm 140$ & $820 \pm 280$ & $940 \pm 280$ \\
\hline
\end{tabular}

s.d. = standard deviation

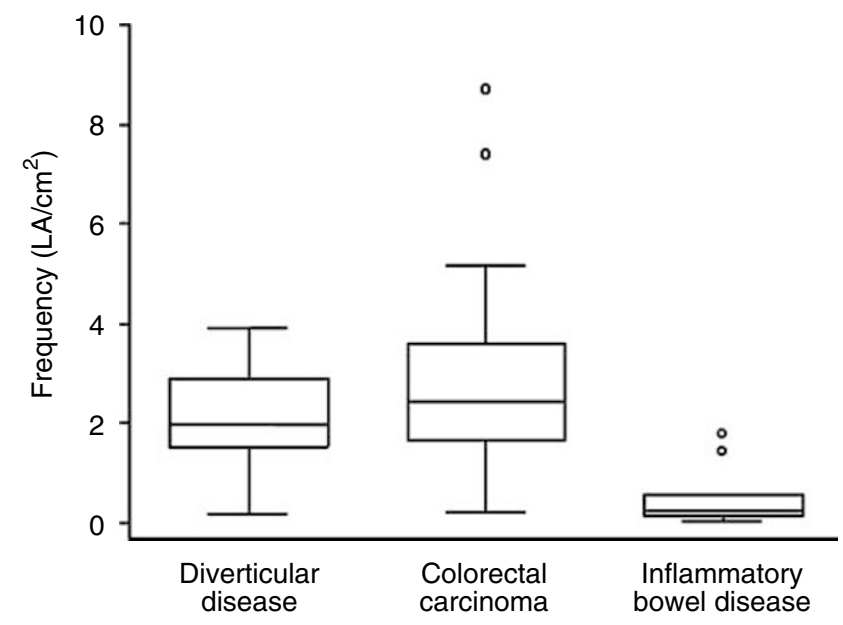

Figure 1 Box and whisker plot showing the median and the distribution of lymphoid aggregate (LA) frequency in normalappearing colorectal specimens after acetic acid staining.

mucosa of patients with ulcerative colitis or with Crohn's disease $(P<0.001)$. No significant difference of density was found by comparing colorectal cancer group with diverticular disease, and Crohn's disease group with ulcerative colitis group.

Neither the difference between lymphoid aggregate frequencies in the right and in the left colon-rectum, nor the difference between frequencies in the colon and in the rectum were statistically significant. Site analysis was conducted in specimens from colorectal cancer patients only, given the small sample size in the other disease groups.

Lymphoid aggregate diameter (Figure 2) was smaller in patients with diverticular disease than in those with carcinoma and those with ulcerative colitis or Crohn's disease $(P<0.001)$. No difference was found by comparing the colorectal cancer group, the Crohn's disease group and the ulcerative colitis group.

Among patients with colorectal cancer, neither the difference between lymphoid aggregate diameter in the right colon and in the left colon and rectum, nor the difference between aggregate diameter in the colon and in the rectum were statistically significant.

The mean diameter did not vary according to patient gender; the diameter in patients aged under 65 years (median) $(790 \pm 210 \mu \mathrm{m})$ was larger than

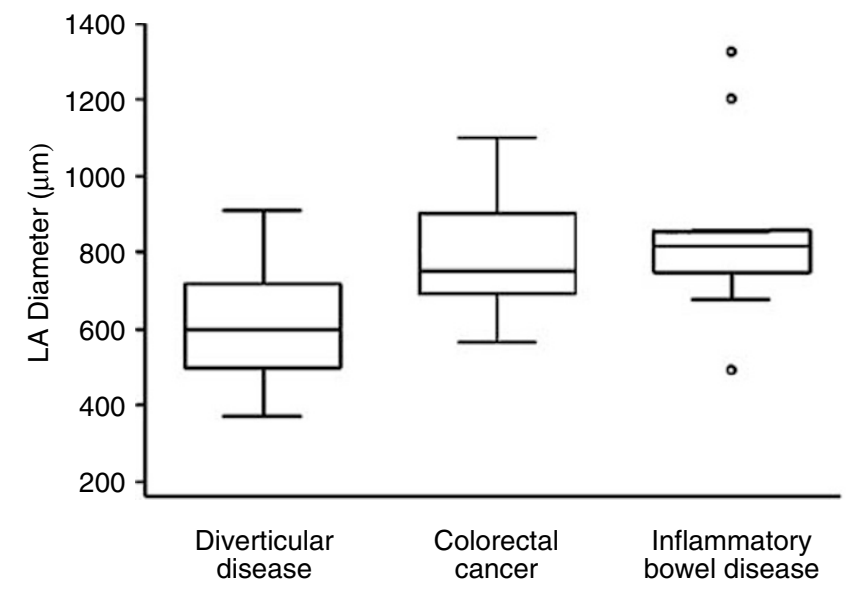

Figure 2 Box and whisker plot showing the median and the distribution of lymphoid aggregate (LA) diameter in normalappearing colorectal specimens after acetic acid staining.

that in patients over 65 years $(690 \pm 160 \mu \mathrm{m})$ $(P=0.03)$.

\section{Histology and Immunohistochemistry}

A total of 111 colorectal specimens (total area $222 \mathrm{~cm}^{2}$ ) were collected for histology and immunohistochemistry. The results of the related assessments are schematized in Table 2 .

Comparison of lymphoid aggregate densities obtained histologically with those obtained with acetic acid staining showed no significant difference between the two methods.

In normal-appearing specimens, the density varied significantly among the four disease groups, again being higher in the mucosa of patients with colorectal cancer or diverticular disease than in the mucosa of patients with ulcerative colitis or with Crohn's disease $(P=0.001)$. In actively affected specimens, the lymphoid aggregate density of patients with ulcerative colitis or with Crohn's disease was significantly higher than that of patients with diverticular disease $(P=0.002)$.

Lymphoid aggregate densities in actively affected specimens were higher than those of normalappearing counterparts in patients with diverticular disease $(P<0.0001)$, with ulcerative colitis $(P=0.004)$, and with Crohn's disease $(P=0.04)$. 
Table 2 Overview of lymphoid aggregate features (H\&E staining)

\begin{tabular}{|c|c|c|c|c|c|c|c|}
\hline & \multirow[t]{2}{*}{ Cancer } & \multicolumn{2}{|c|}{ Diverticular disease } & \multicolumn{2}{|c|}{ Ulcerative colitis } & \multicolumn{2}{|c|}{ Crohn } \\
\hline & & Uninvolved & Active & Uninvolved & Active & Uninvolved & Active \\
\hline Specimen no. & 41 & 27 & 25 & 6 & 6 & 3 & 3 \\
\hline Lymphoid aggregate density & $2.7 \pm 1.2$ & $2.5 \pm 1.1$ & $4.5 \pm 1.7$ & $1.3 \pm 0.6$ & $9.3 \pm 6.3$ & $0.5 \pm 0.5$ & $20.8 \pm 5.2$ \\
\hline Lymphoid aggregates with germinal centers (\%) & $2 \overline{9} .6$ & $2 \overline{9} .6$ & $1 \overline{7} .7$ & $3 \overline{1} .2$ & $2 \overline{5} .0$ & $3 \overline{3} .3$ & $0 . \overline{8}$ \\
\hline Basal aggregates (\%) & 5.0 & 5.9 & 6.6 & 6.3 & 19.6 & 0 & 83.2 \\
\hline CD3-positive aggregates (\%) & 100 & 100 & 100 & 100 & 100 & 100 & 100 \\
\hline CD20-positive aggregates (\%) & 93.5 & 93.5 & 91.6 & 92.5 & 90.8 & 91.7 & 91.7 \\
\hline MIB-1 positive & $5: 02$ & 5:01 & $5: 06$ & $5: 03$ & 7:05 & $5: 00$ & $5: 00$ \\
\hline
\end{tabular}

Lymphoid aggregate density $=$ mean aggregate count $/ \mathrm{cm}^{2} \pm$ s.d.

The proportion of aggregates with germinal center in normal-appearing specimens was similar among the four disease groups, while in actively affected colorectal samples it was higher in ulcerative colitis and diverticular disease than in Crohn's disease $(P=0.002)$. The proportion of aggregates with germinal center in actively affected specimens was lower than those of normal-appearing counterparts in patients with diverticular disease $(P<0.0001)$, while no significant difference was found in patients with ulcerative colitis, or with Crohn's disease.

The proportion of basal lymphoid aggregates in normal-appearing specimens was similar among the four disease groups, while in actively affected colorectal samples it was higher in Crohn's disease and in ulcerative colitis than in diverticular disease $(P<0.0001)$. The proportion of basal aggregates in actively affected specimens was higher than those of normal-appearing counterparts in patients with ulcerative colitis $(P=0.008)$, and with Crohn's disease $(P=0.04)$, while no significant difference in patients with diverticular disease.

Neither among the four disease groups nor between actively affected and uninvolved specimens, there was any difference in lymphocyte distribution. Predominantly B cells were concentrated in the central area of the aggregate whereas the $\mathrm{T}$ cells constituted the peripheral shell. B lymphocyte were not found in up to $9.2 \%$ of lymphoid aggregates possibly reflecting sampling defects.

Neither among the four disease groups nor between actively affected and uninvolved specimens, there was any difference in lymphocyte proliferation index assessed by MIB-1 activity.

\section{Discussion}

The little available information on morphological characteristics of colorectal lymphoid aggregates is dated and controversial.

A major drawback of previous studies is the employment of different methods to conduct lymphoid aggregate assessment. ${ }^{1-4}$ The comparable density obtained with acetic acid staining and with H\&E histology in our study suggest that the latter method is reliable, reproducible, and permits to evaluate the lymphoid aggregate density as well as additional parameters in conditions of active inflammation also.

A wide range of 'normal' densities (from 3 to 25 aggregates $/ \mathrm{cm}^{2}$ ) has been obtained in necropsy specimens, ${ }^{1-3}$ whereas the data on 'normal' diameter are scarce, being limited to an approximate range between 0.5 and $2 \mathrm{~mm}^{2,3}$ The reliability of both figures remains debatable since they are obtained in a limited number of cases and using different methods. No definite new information is added to this issue by our study since all specimens were collected from living patients with colorectal diseases. The mean density found in the unaffected bowel of patients with colorectal cancer or diverticular disease, using two assessment methods, however, may conceivably reflect the mean lymphoid aggregate density of healthy individuals. Accordingly, it should range from 2.1 to 2.7 aggregates $/ \mathrm{cm}^{2}$.

The involvement of lymphoid aggregates in colorectal carcinogenesis is debated. Data from both rodents and humans have shown a coincidence of colorectal neoplasms with aggregate location. ${ }^{10-15}$ Moreover, increased lymphoid aggregate densities in patients with colorectal carcinoma have been reported by morphologic ${ }^{2}$ and radiographic studies. ${ }^{8}$

Discussing the potential role of lymphoid aggregates on colorectal carcinogenesis is beyond the limit of the present study. However, we did not find any evidence of increased aggregate density in colorectal cancer group, and our data indicate that a casual proximity of adenomas or carcinomas with lymphoid aggregates is likely $(1 \mathrm{~cm}$ sessile lesion lies on an average of 2.7 aggregates).

In diverticular disease, mean aggregate density has been reported to range between 5.8 aggregates/ $\mathrm{cm}^{24}$ and 21 aggregates $/ \mathrm{cm}^{2}{ }^{2}$ Unfortunately, none of the available studies did disclose whether tissue sampling was performed close to inflamed diverticula or far from them. By comparing both conditions, our study shows that active diverticular inflammation results in a double-fold increase of lymphoid aggregate density. Diverticulitis-induced 
lymphoid aggregates maintain their subepithelial location and lymphocyte composition, although an higher proportion of them is constituted by follicles without germinal center. Possibly, the alterations seen closed to inflamed diverticula constitute the standard colorectal lymphoid tissue rearrangement in response to nonspecific inflammatory stimuli.

A number of studies have investigated the role of circulating and mucosal immune cells in the pathogenesis and evolution of inflammatory bowel diseases. Data on morphologic features of lymphoid aggregates are, however, scarce and limited to the actively inflamed colorectum.

In the actively involved specimens of patients with ulcerative colitis or Crohn's disease, our data confirmed the previously noted modifications of lymphoid aggregate system. ${ }^{7,16,17}$ The increase of density in active inflammatory bowel diseases exceeds by two to four folds the aggregate proliferation seen close to inflamed diverticula. Moreover, most of aggregates in Crohn's disease and a substantial part of them in ulcerative colitis is positioned deeply in the submucosa or even transmurally. Hence, the rearrangement of lymphoid aggregate system in active inflammatory bowel diseases differs widely from the simple subepithelial aggregate proliferation seen in diverticular disease. In order to evaluate the role of this anomalous response in the pathogenesis of inflammatory bowel diseases, we believe, however, it is crucial to verify any derangement of the lymphoid aggregate system eventually pre-existing in the uninvolved colorectal mucosa.

The uninvolved mucosa in Crohn's disease and ulcerative colitis received little attention in the past, being considered normal macroscopically and microscopically. ${ }^{18}$ We have compared the lymphoid aggregate characteristics in normalappearing mucosa of patients with Crohn's disease or ulcerative colitis with those in normal-appearing specimens of patients with colorectal cancer or diverticulitis. A five-fold and a two-fold reduction of aggregate density have been found in Crohn's disease and in ulcerative colitis, respectively. Moreover, a marked increase of aggregate diameter was seen especially in Crohn's disease. On the other hand, the distribution of $\mathrm{B}$ and $\mathrm{T}$ lymphocytes was unaffected by the presence or absence of active disease involvement.

Previous studies have suggested that Peyer's patches may be involved in the pathogenesis of Crohn's disease. ${ }^{19}$ We found that the lymphoid aggregate component of mucosa-associated lymphoid tissue is altered even in the uninvolved colorectal lining of inflammatory bowel diseases. Our finding supports a primary pathogenic involvement of the lymphoid aggregate system in Crohn's disease and in ulcerative colitis, since it suggests the presence of an altered mucosal substrate prone to extension or to relapse of the inflammatory disease.

\section{References}

1 Dukes C, Bussey HJR. The number of lymphoid follicles of the human large intestine. J Pathol Bacteriol 1926;29:111-116.

2 O'Learly AD, Sweeney EC. Lymphoglandular complexes of the colon: structure and distribution. Histopathology 1986;10:267-283.

3 Langman JM, Rowland R. The number and distribution of lymphoid follicles in the human large intestine. J Anat 1986;194:189-194.

4 Kealy WF. Lymphoid tissue and lymphoid-glandular complexes of the colon: relation to diverticulosis. J Clin Pathol 1976;29:245-249.

5 Whelan RL, Abramson D, Kim DS, et al. Diversion colitis. A prospective study. Surg Endosc 1994;8: 19-24.

6 Washington K, Stenzel TT, Buckley RH, et al. Gastrointestinal pathology in patients with common variable immunodeficiency and X-linked agammaglobulinemia. Am J Surg Pathol 1996;20:1240-1252.

7 Tanaka M, Riddell RH. The pathological diagnosis and differential diagnosis of Crohn's disease. Hepatogastroenterology 1990;37:18-31.

8 Glick SN, Teplcik SK, Ross WM. Colonic lymphoid follicles associated with colonic neoplasms. Radiology 1988;168:603-607.

9 Bharadhway G, Triadafilopoulos G. Endoscopic appearances of colonic lymphoid nodules: new faces of an old histopathological entity. Am J Gastroenterol 1995;90:946-950.

10 Carter JW, Lancaster HK, Hardman WE, et al. Distribution of intestine lymphoid tissue, aberrant crypt foci and tumours in the large bowel of 1,2-dimethylhidrazine-treated mice. Cancer Res 1994;54:4304-4307.

11 Cameron IL, Garza J, Hardman WE. Distribution of lymphoid nodules, aberrant crypt foci and tumours in the colon of carcinogen-treated rats. $\mathrm{Br} \mathrm{J}$ Cancer 1996;73:893-898.

12 Rubio CA, Shetye J, Jaramillo E. Non-polypoid adenomas of the colon are associated with subjacent lymphoid nodules. Scand J Gastroenterol 1999;5: 504-508.

13 Oohara T, Ogino A, Tohma H. Microscopic adenoma in nonpolyposis coli: incidence and relation to basal cells and lymphoid follicles. Dis Colon Rectum 1981;24: 120-126.

14 Shpitz D, Bomstein Y, Mekori Y, et al. Aberrant crypt foci in human colons: distribution and histomorphologic characteristics. Human Pathol 1998;29:469-475.

15 Nascimbeni R, Di Betta E, Villanacci V, et al. Association of lymphoid aggregates with aberrant crypt foci in human colon. Gastroenterology 1998;114:G2689.

16 Le Berre N, Heresbach D, Kerbaol M, et al. Histological discrimination of idiopathic inflammatory bowel disease from other types of colitis. J Clin Pathol 1995;48: 749-753.

17 Yeung MM, Melgar S, Baranov V, et al. Characterisation of mucosal lymphoid aggregates in ulcerative colitis: immune cell phenotype and TcR-gammadelta expression. Gut 2000;47:215-227.

18 Goodman MJ, Skinner JM, Truelove SC. Abnormalities in the apparently normal bowel mucosa in Crohn's disease. Lancet 1976;1:275-278.

19 Van Kruiningen HJ, Ganley LM, Freda BJ. The role of Peyer's patches in the age-related incidence of Crohn's disease. J Clin Gastroenterol 1997;24:470-475. 